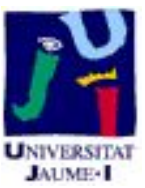

Título artículo / Títol article: Importance of MS selectivity and chromatographic separation in LC-MS/MS-based methods when investigating pharmaceutical metabolites in water. Dipyrone as a case of study

Autores / Autors

M. Ibáñez, E. Gracia-Lor, J. V. Sancho, F. Hernández

Journal of Mass Spectrometry, 47 (2012) p. 1040-1046

Revista:

Versión / Versió: $\quad$ Versión pre-print del autor

Cita bibliográfica / Cita

IBÁÑEZ, María, et al. Importance of MS selectivity and bibliogràfica (ISO 690): chromatographic separation in LC-MS/MS-based methods when investigating pharmaceutical metabolites in water. Dipyrone as a case of study. Journal of Mass Spectrometry, 2012, vol. 47, no 8, p. 1040-1046.

url Repositori UJI:

http://hdl.handle.net/10234/60984 


\title{
Importance of MS selectivity and chromatographic separation in LC-MS/MS-based methods when investigating pharmaceutical metabolites in water. Dipyrone as a case of study
}

\author{
Journal of Mass Spectrometry, 47 (2012) 1040-1046 \\ M. Ibáñez, E. Gracia-Lor, J. V. Sancho and F. Hernández \\ Research Institute for Pesticides and Water, University Jaume, Castellón, Spain
}

\begin{abstract}
Pharmaceuticals are emerging contaminants of increasing concern because of their presence in the aquatic environment and potential to reach drinking-water sources. After human and/or veterinary consumption, pharmaceuticals can be excreted in unchanged form, as the parent compound, and/or as free or conjugated metabolites. Determination of most pharmaceuticals and metabolites in the environment is commonly made by liquid chromatography (LC) coupled to mass spectrometry (MS). LC coupled to tandem MS is the technique of choice nowadays in this field. The acquisition of two selected reaction monitoring (SRM) transitions together with the retention time is the most widely accepted criterion for a safe quantification and confirmation assay. However, scarce attention is normally paid to the selectivity of the selected transitions as well as to the chromatographic separation. In this work, the importance of full spectrum acquisition high-resolution MS data using a hybrid quadrupole time-of-flight analyser and/or a suitable chromatographic separation (to reduce the possibility of co-eluting interferences) is highlighted when investigating pharmaceutical metabolites that share common fragment ions. For this purpose, the analytical challenge associated to the determination of metabolites of the widely used analgesic dipyrone (also known as metamizol) in urban wastewater is discussed. Examples are given on the possibilities of reporting false positives of dypirone metabolites by LC-MS/MS under SRM mode due to a wrong assignment of identity of the compounds detected.
\end{abstract}

\section{Keywords}

Dipyrone; Pharmaceutical metabolites; Ultrahigh pressure liquid chromatography; Timeof-flight mass spectrometry; $\mathrm{MS}^{\mathrm{E}}$; Urban wastewater; False positives. 


\section{Introduction}

The presence of pharmaceuticals in the environment is a matter of concern because of their wide consumption and potential negative effect on the water quality and living organisms. ${ }^{[1,2]}$ After human and/or veterinary consumption, pharmaceuticals are excreted in unchanged form, as the parent compound, and also as free and/or conjugates metabolites. Once pharmaceuticals reach the aquatic environment, both the parent compound and metabolites can suffer different transformation processes, mainly photodegradation and hydrolysis, producing a variety of transformation products in water.

Most recent methods for the determination of pharmaceuticals in the environment are based on the use of liquid chromatography coupled to mass spectrometry (LC-MS), and particularly on LC-tandem mass spectrometry (LC-MS/MS) using triple quadrupole (QqQ) analyser. $^{[3,4]}$ The usual approach has been the acquisition of two transitions in LC-MS/MS methods, which fulfills existing guidelines. ${ }^{[5,6]}$ Confirmation of positive findings is based on the accomplishment of ion ratio and retention time, which must fit the limits established by current guideliness. Under these circumstances, not much attention is paid to the chromatographic separation and/or transitions selectivity, assuming that other compounds present in the sample will not fulfil these criteria.

The satisfactory sensitivity in full spectrum acquisition mode, high resolution (HR), exact mass measurements and MS/MS capabilities of hybrid quadrupole time-of-flight (QTOF) mass spectrometry make of this technique a powerful analytical tool for the identification and confirmation of organic contaminants. Moreover, and opposed to analytical methods based on selected ion monitoring or selected reaction monitoring (SRM), accurate-mass full-spectrum data generated by LC-(Q)TOF MS remain available over time. This allows investigating any other compound in addition to target analytes, provided such compound has passed the sample preparation, chromatographic separation and ionization process with sufficient efficiency. This fact clearly represents an important advantage of HRMS for wide scope screening of organic contaminants. ${ }^{[7-9]}$ In the last years, there has been a growing trend on using TOF MS for screening and confirmation of pharmaceuticals in environmental samples. ${ }^{[4,10-12]}$ However, it has been scarcely used to investigate pharmaceutical metabolites in the environment. ${ }^{[13]}$

Dipyrone (DIP, also known as metamizol) is a non-steroidal anti-inflammatory drug, commonly used to treat severe pain associated with colic, cancer and migranes. ${ }^{[14]}$ It is marketed under various trade names, including Conme $/ \AA$, Neo-Melubrina $\AA$, Magnopyro $(\AA$, Prodolina $F \circledast$, Analgin $\AA$, Nolotil $($ and Novalgin $\AA$. Immediately after its introduction in 1922 , the use of DIP was linked to serious toxic effects, such as dose-independent teratogenicity, increased bleeding times and a potentially fatal agranulocytosis. ${ }^{[15]}$ While the use of DIP has been banned in USA, Canada or UK, in many Western European countries (e.g. Germany, Italy or Spain), it is still one of the most consumed pharmaceuticals. ${ }^{[16]}$ DIP is considered a pro-drug. After oral intake, it is rapidly hydrolyzed to 4-methylaminoantipyrine (4-MAA), which is absorbed 
and bio-transformed by enzymatic reactions. In the liver, 4-MAA is metabolized to 4aminoantipyrine (4-AA) via demethylation and further acetylated to 4-acetylaminoantipyrine (4AAA). Another metabolite, 4-formylaminoantipyrine (4-FAA), is generated by oxidation of the methyl group. ${ }^{[16]}$ The presence of these DIP metabolites has been reported in sewage treatment plant effluents ${ }^{[12,17]}$ and in surface water. ${ }^{[17]}$

In a previous work, ${ }^{[18]}$ the presence of 4-amino antipyrine (4-AA) in urban wastewater was reported based on the monitoring of two SRM transitions using a LC-MS/MS method. A slight shift in retention time together with the presence of clear shoulders in the chromatographic peak was observed. However, both retention time and ion ratio deviations as regards the reference standard were within the tolerances established. Despite all requirements established by current guidelines were satisfied, reasonable doubts about a false positive due to unresolved interferences might be hypothesized. The goal of this paper is to point out the benefits of using full spectrum acquisition HRMS, and the need of applying efficient chromatographic separation in some particular complex situations, as for example when investigating pharmaceutical metabolites. For this purpose, the analytical challenge associated to the determination of DIP metabolites in urban wastewater is studied in depth making use of QTOF and QqQ analysers both coupled to UPLC.

\section{Experimental}

\subsection{Reagents and chemicals}

Reference standards (purity higher than 93\%) of 4-AA, 4-AAA and 4-FAA were purchased from Sigma-Aldrich (St Louis, MO, USA) and Toronto Research Chemicals (Ontario, Canada).

HPLC-grade water was obtained by purifying demineralised water in a Milli-Q plus system from Millipore (Bedford, MA, USA). HPLC-grade methanol (MeOH), Sodium hydroxide $>99 \%(\mathrm{NaOH})$, ammonia solution (25\%), and formic acid (98-100\%) were acquired from Scharlau (Barcelona, Spain). Leucine encephalin (used as the lock mass) was purchased from Sigma-Aldrich.

\subsection{Instrumentation}

\section{UHPLC-QTOF MS}

A Waters Acquity UPLC system (Waters, Milford, MA, USA) was interfaced to a hybrid quadrupole-orthogonal acceleration-TOF mass spectrometer (XEVO G2 QTOF, Waters Micromass, Manchester, UK), using an orthogonal Z-spray-ESI interface operating in positive ion mode. The chromatographic separation was performed using an Acquity UPLC BEH C18 
1.7- $\mu \mathrm{m}$ particle size analytical column $100 \times 2.1 \mathrm{~mm}$ (Waters) at a flow rate of $300 \mu \mathrm{L} / \mathrm{min}$. The mobile phases used were $A=\mathrm{H}_{2} \mathrm{O}$ with $0.01 \% \mathrm{HCOOH}$ and $\mathrm{B}=\mathrm{MeOH}$ with $0.01 \% \mathrm{HCOOH}$. The initial percentage of $B$ was $10 \%$, which was linearly increased to $90 \%$ in $14 \mathrm{~min}$, followed by a 2 min isocratic period and, then, returned to initial conditions during $2 \mathrm{~min}$ in total run duration of $18 \mathrm{~min}$. Nitrogen was used as the drying gas and nebulizing gas. The gas flow was set at $1200 \mathrm{~L} / \mathrm{h}$. TOF-MS resolution was approximately 25000 at full width half maximum at $\mathrm{m} / \mathrm{z} 556$. MS data were acquired over an $\mathrm{m} / \mathrm{z}$ range of $50-1200$. A capillary voltage of $0.7 \mathrm{kV}$ and cone voltage of $20 \mathrm{~V}$ were used. Collision gas was argon $99.995 \%$ (Praxair, Valencia, Spain). The interface temperature was set to $600^{\circ} \mathrm{C}$ and the source temperature to $120^{\circ} \mathrm{C}$. The column temperature was set to $40^{\circ} \mathrm{C}$.

For $\mathrm{MS}^{\mathrm{E}}$ experiments, two acquisition functions with different collision energies were created. The low energy function (LE), selecting a collision energy of $4 \mathrm{eV}$, and the high energy (HE) function, with a collision energy ramp ranging from 15 to $40 \mathrm{eV}$ in order to obtain a greater range of fragment ions. The LE and HE functions settings were for both a scan time of $0.3 \mathrm{~s}$.

Calibrations were automatically conducted from $\mathrm{m} / \mathrm{z} 50$ to 1200 with a $1: 1$ mixture of $0.05-\mathrm{M} \mathrm{NaOH}: 5 \% \mathrm{HCOOH}$ diluted (1:25) with acetonitrile:water (80:20). For automated accurate mass measurement, the lock-spray probe was used, using as lockmass a solution of leucine enkephalin $(2 \mu \mathrm{g} / \mathrm{mL})$ in acetonitrile:water (50:50) at $0.1 \% \mathrm{HCOOH}$ pumped at $20 \mu \mathrm{L} / \mathrm{min} . \mathrm{m} / \mathrm{z}$ 556.2771 and $\mathrm{m} / \mathrm{z} 278.1141$ (corresponding to the protonated molecule of leucine enkephalin and to a fragment ion, respectively) were used for recalibrating the mass axis and ensuring a robust accurate mass measurement along time.

\section{UPLC-MS/MS}

For MS/MS experiments on the triple quadrupole (TQD) mass spectrometer, chromatographic separation was performed using an Acquity UPLC HSS T3 column $\left(\mathrm{C}_{18}\right)$, $1.8 \mu \mathrm{m}, 100 \times 2.1 \mathrm{~mm}$ (Waters) at a flow rate of $300 \mu \mathrm{L} / \mathrm{min}$. Mobile phase consisted of a water/methanol, both $0.1-\mathrm{mM} \mathrm{NH}_{4} \mathrm{Ac}$ and $0.01 \% \mathrm{HCOOH}$, gradient. The methanol percentage was changed linearly as follows: $0 \mathrm{~min}, 5 \% ; 5 \mathrm{~min}, 90 \% ; 6 \mathrm{~min}, 90 \% ; 6.1 \mathrm{~min} ; 5 \%$. Analysis run time was $10 \mathrm{~min}$. The sample injection volume was $20 \mu \mathrm{L}$. A TQD mass spectrometer with an orthogonal Z-spray-electrospray interface (Waters) was used. Drying gas as well as nebulising gas was nitrogen generated from pressurized air in a N2 LC-MS (Claind, Teknokroma, Barcelona, Spain). The cone gas and the desolvation gas flows were set at $60 \mathrm{~L} / \mathrm{h}$ and $1200 \mathrm{~L} / \mathrm{h}$, respectively. For operation in MS/MS mode, collision gas was Argon 99.995\% (Praxair, Valencia, Spain) with a performance of $2 \times 10-3 \mathrm{mbar}$ in the T-Wave collision cell. A capillary voltage of $3.5 \mathrm{kV}$ (positive ionization mode) and a cone voltage of $20 \mathrm{~V}$ were applied. The interface temperature was set to $500^{\circ} \mathrm{C}$, and the source temperature to $120^{\circ} \mathrm{C}$. A dwell time of $0.01 \mathrm{~s}$ was selected. Selected SRM transitions were the following: $204.4>56.1$ and $204.4>83.1$ 
for $4-A A, 232.4>56.1$ and $232.4>214.3$ for $4-F A A$ and, $246.4>83.1$ and $246.4>228.3$ for 4 AAA.

\subsection{Water samples}

Twenty-four effluent wastewaters extracts from the Spanish Mediterranean area, previously analysed by UHPLC-QqQ MS after being subjected to solid-phase extraction with Oasis HLB (60 mg), ${ }^{[18]}$ were re-injected by UHPLC-(Q)TOF MS in order to investigate the possible presence of 4-AA and other metabolites of DIP.

\section{Results and discussion}

In a previous work, we developed a target method ${ }^{[18]}$ based on UHPLC-MS/MS with TQD that was applied to the determination of 45 pharmaceuticals in water. As scientific literature shows, pharmaceutical metabolites have hardly been studied in environmental water and wastewater. Typically, only metabolites of those compounds classified as pro-drugs have been included in target methods. ${ }^{[19-21]}$ This is the case of 4-AA (the main reported metabolite of DIP) and salicylic acid (the main metabolite of acetylsalicylic acid) that were also included in our previous work. $^{[18]} 4-\mathrm{AA}$ was found in around $80 \%$ of the effluent wastewater samples analysed, with a maximum concentration of $2.8 \mu \mathrm{g} / \mathrm{L}$. The two transitions selected, one for quantification (Q) $204>56$, and the other for confirmation (q) $204>83$, were observed in the samples and Q/q ratios were within the maximum deviations admitted. Under the chromatographic conditions employed, a poor chromatographic peak shape was observed in most of samples, presenting a clear shoulder, although the criteria used for confirmation of positives were accomplished. Therefore, the presence of 4-AA was assumed in the samples.

However, the shoulder observed in the LC-MS/MS chromatograms for the two transitions monitored encouraged us to investigate the presence of this metabolite in more detail to discard a false positive. The same water samples extracts were subsequently analysed by UHPLC-(Q)TOF MS under MS ${ }^{\mathrm{E}}$ mode. With $\mathrm{MS}^{\mathrm{E}}$ experiments, both (de)protonated molecule and fragment ion data are enabled in a single acquisition, without the need of selecting the precursor ion, a notable difference with true MS/MS experiments. ${ }^{[8,22,23]}$

Figure 1 shows LE and HE spectra of the 4-AA reference standard. The elemental composition for up to seven main fragments observed in the HE TOF MS spectrum was calculated, obtaining errors for accurate masses normally below $0.5 \mathrm{mDa}$ in relation to the theoretical predicted exact masses. The structures suggested by MassFragment software are 
also depicted, showing that the product ions previously selected for SRM transitions $(\mathrm{m} / \mathrm{z} 56$ and 83) seem selective enough.
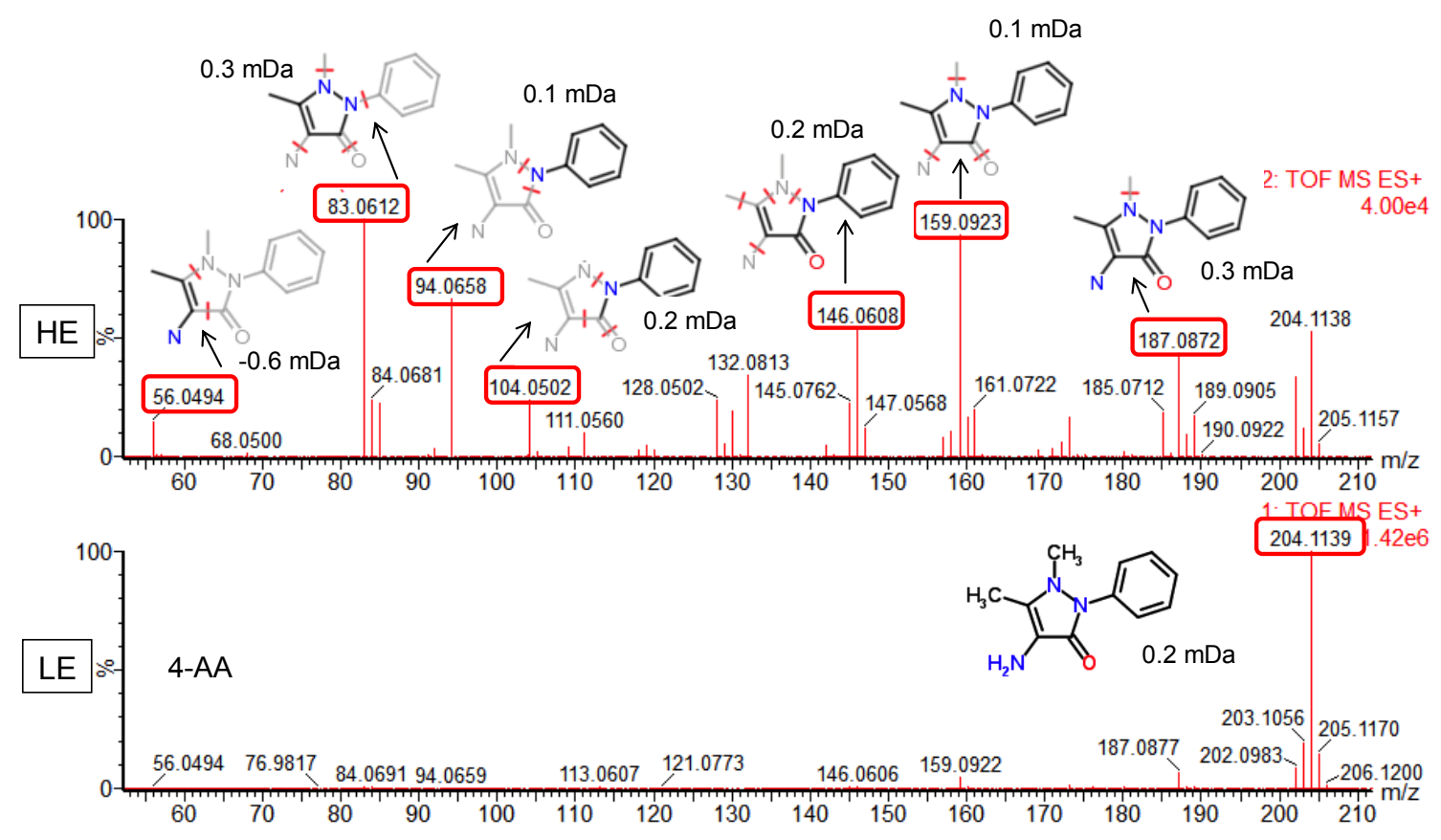

Figure 1. LE and HE spectra of 4-aminoantipyrine (4-AA). Structures proposed by MassFragment software for fragment ions of this compound.

In the samples analysed by QTOF MS, the narrow-window extracted ion chromatograms (nw-XIC) $(20-\mathrm{mDa} \text { mass window })^{[24,25]}$ at the exact mass of 4-AA protonated molecule $\left(\mathrm{m} / \mathrm{z}[\mathrm{M}+\mathrm{H}]^{+}\right.$204.1137) were obtained from LE MS data. In several samples, two close peaks were observed, as shown in Fig. 2a. The chromatographic peak at 3.33 min was assigned, in principle, to 4-AA as the accurate mass ( $\mathrm{m} / \mathrm{z} 204.1132$, Fig. $2 \mathrm{~b}$ ) differed $-0.5 \mathrm{mDa}$ from the theoretical mass of $4-\mathrm{AA}$, and its retention time did not exceed the maximum deviation of $2.5 \%$ typically allowed in LC-MS (reference retention time, $3.25 \mathrm{~min}$ ). However, the accurate mass of the second peak, at $3.45 \mathrm{~min}(\mathrm{~m} / \mathrm{z} 204.1142$, Fig. 2c) also differed only $0.5 \mathrm{mDa}$ from the theoretical mass of 4-AA. When performing the nw-XICs at the expected exact masses of 4AA fragment ions (HE function), two chromatographic peaks were observed in each case at $\mathrm{m} / \mathrm{z}$ 159, 146, 104, 83 and 56, at both retention times (Fig. 2a). This fact strongly supported that the identity of the compound at 3.33 min was $4-A A$. Obviously, the other peak at 3.45 min should correspond to a highly related compound. 
However, the mass spectra of the compound eluting at 3.33 min presented two differentiated ions in the LE spectrum, at $\mathrm{m} / \mathrm{z}$ values higher than the 4-AA protonated molecule. One of those was a highly abundant ion at $\mathrm{m} / \mathrm{z} 232.1088$ (Fig. 2b), and the other was a fragment ion at $m / z$ 214.0982. The later was more visible in the HE spectrum $(m / z 214.0976)$. The $n w-$ $X I C s$ at $m / z 232.1088$ for the LE and $m / z 214.0976$ for the HE function showed the presence of peaks at the same retention time with the same peak shape (Fig. 2a). Thus, it seemed that the accurate mass of the protonated molecule for this compound was 232.1088, and not 204.1132 as initially believed. In addition, the $\mathrm{m} / \mathrm{z} 214.0976$ ion could be explained as a fragment derived from 232 due to a water loss. Obviously, the compound detected was not 4-AA, but it should be related with it as both shared up to six fragment ions. After searching in available database, ${ }^{[13]}$ this compound was identified as 4-FAA.

In a subsequent step, the reference standard was acquired and injected, confirming the identity of this compound. Hence, the presence of 4-AA was wrongly assigned when this sample was analysed by LC-MS/MS QqQ using the transitions $204>56$ (Q) and $204>83$ (q). It seems that 4-FAA produced an in-source fragment at $\mathrm{m} / \mathrm{z} 204$, which was afterwards isolated and fragmented in the collision cell to give the product ions at $\mathrm{m} / \mathrm{z} 56$ and 83 . In addition, both compounds eluted in our chromatographic system at nearly the same retention time, making likely the reporting of 4-AA false positives. The occurrence of 4-FAA in wastewater has been previously investigated by Martínez-Bueno et al. ${ }^{[12]}$ This metabolite was detected in 14 out of the 19 samples analysed, at concentrations between 0.04 and $10 \mu \mathrm{g} / \mathrm{L}$.

Regarding the peak at $3.45 \mathrm{~min}$, the LE spectrum presented an abundant ion at $\mathrm{m} / \mathrm{z}$ 246.1252 and the HE spectra at $\mathrm{m} / \mathrm{z} 228.1137$ (also corresponding to a water loss) (Fig. 2c). The nw-XICs at these masses showed the presence of a chromatographic peak at the same retention time (3.45 min) (Fig. 2a). Again, it seemed that this compound was closely related to 4$A A$, as both shared up to six fragment ions. After searching in available database ${ }^{[13]}$ this compound was identified as 4-AAA. As before, the reference standard was subsequently acquired and injected, confirming the identity of the compound. This metabolite has been also reported in wastewater at concentrations between 2 and $25 \mu \mathrm{g} / \mathrm{L}^{[12]}$ 


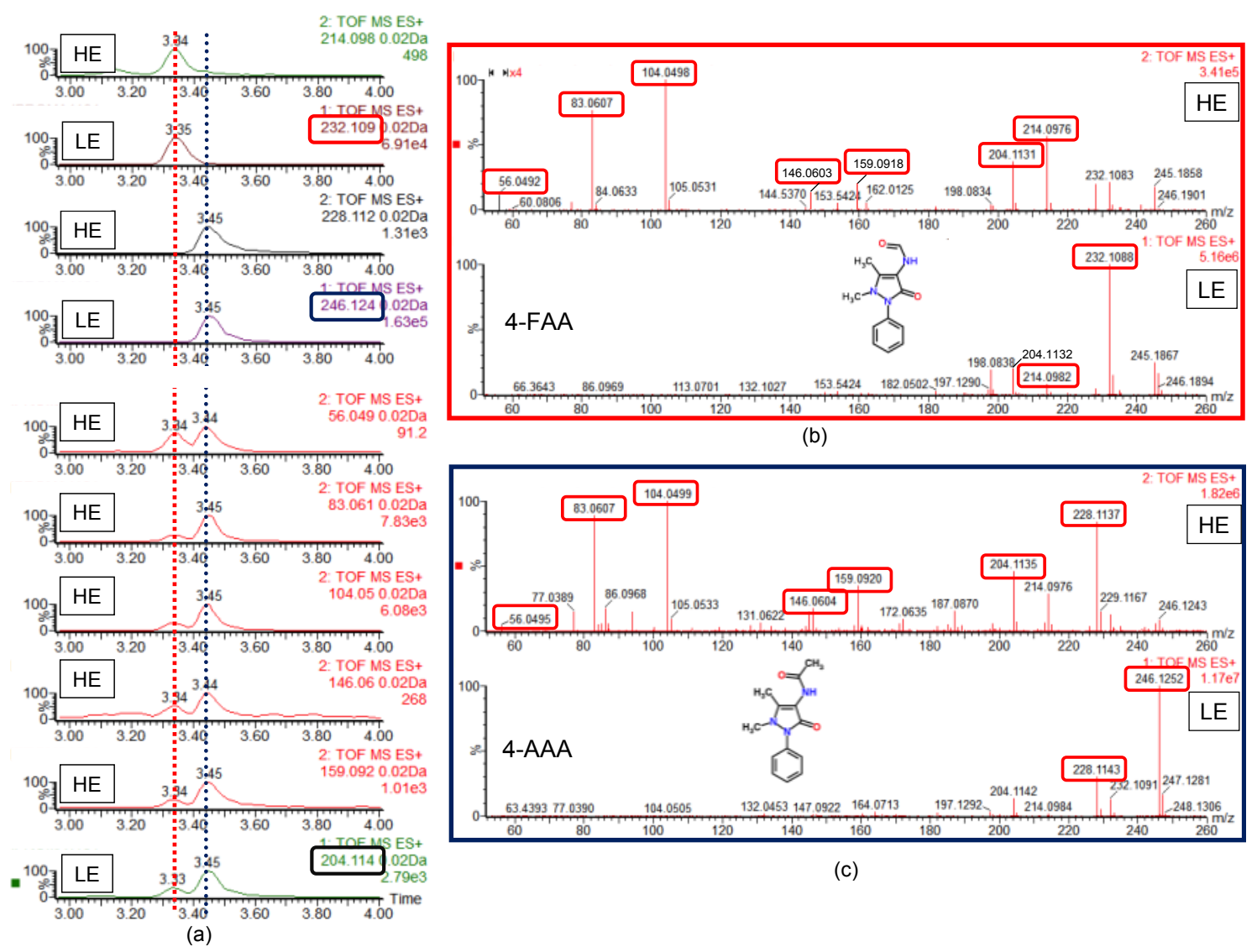

Figure 2. Detection and identification of 4-formylaminoantipyrine (4-FAA) and 4acetylaminoantipyrine (4-AAA) in an effluent wastewater by UHPLC-QTOF under MS ${ }^{E}$ mode: (a) XICs (20-mDa mass window) for $[\mathrm{M}+\mathrm{H}]^{+}$in LE function and for the main fragments in $\mathrm{HE}$ function; (b) LE and HE spectra of 4-FAA in the sample (chromatographic peak at $3.33 \mathrm{~min}$ ); (c) LE and HE spectra of 4-AAA in the sample (chromatographic peak at $3.45 \mathrm{~min}$ ).

Thus, in this particular water sample, the metabolite detected was not 4-AA, but 4-AAA and 4-FAA. A false positive of 4-AA would have been reported if they were not chromatographically resolved. Under the chromatographic conditions employed in the UPLCQTOF MS, the three metabolites were roughly resolved as all eluted at close retention times. As illustrative example, Fig. 3 shows the UPLC-TOF MS chromatograms for a wastewater sample that was positive for the three metabolites, and where the presence of 4-AA could be unambiguously reported preventing its false identification. However, if no efficient chromatographic separation occurs for these metabolites, the presence of 4-AA cannot be ensured, even using HR in MS/MS mode, as derived from Müller et al. ${ }^{[26]}$ In that paper, 4-AA and 4-AAA perfectly co-eluted at $6.0 \mathrm{~min}$. As 4-AA shares all its product ions with 4-AAA, the detection of the ion at $\mathrm{m} / \mathrm{z} 204$ might be related with an in-source fragment of 4-AAA and not with the presence of 4-AA. Therefore, to prevent such possibility, it is crucial an improvement in the chromatographic separation for minimising false identifications. 

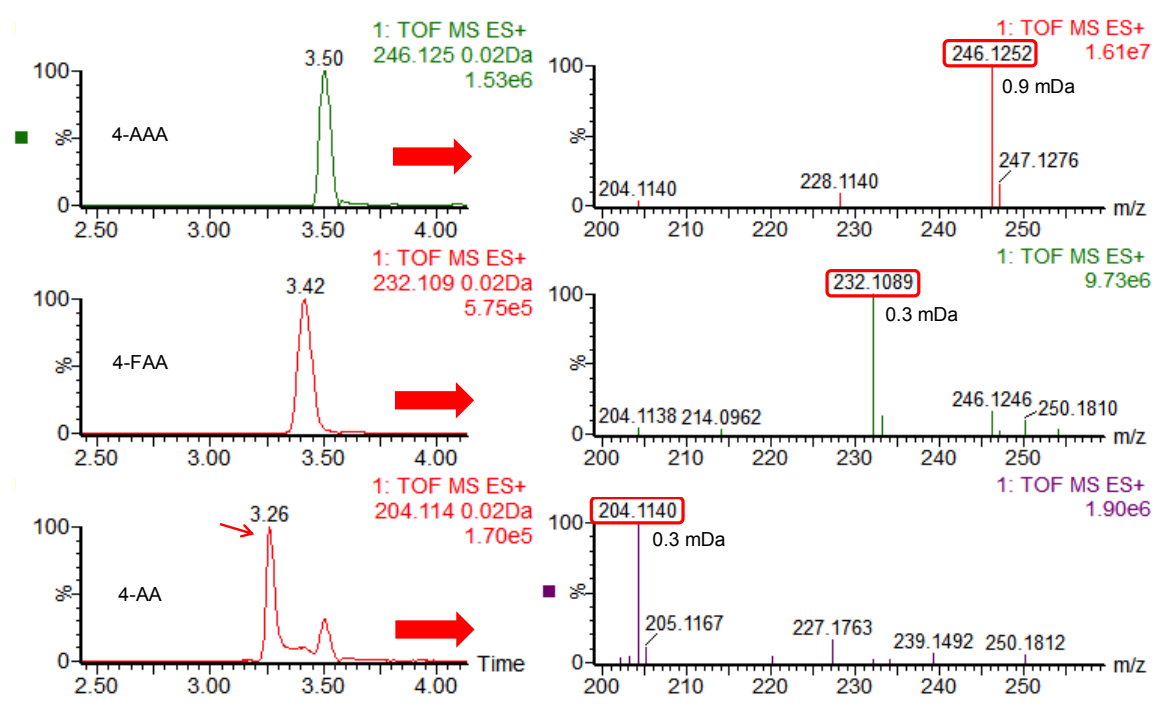

Figure 3. UHPLC-QTOF MS XICs chromatograms and LE spectra for a wastewater sample containing 4-AA, 4-FAA and 4-AAA.

On the basis of these results, the sample extracts previously analysed were injected again in the LC-MS/MS system, with a slight modification in the gradient slope and also considering the transitions for the two additional metabolites identified by TOF MS. Regarding the transitions selected for 4-AA, the cone voltage was reduced down to $20 \mathrm{~V}$, to minimise insource fragmentation of the other metabolites. Figure 4 shows UHPLC-MS/MS chromatograms for a reference standard containing 4-AA, 4-AAA and 4-FAA, as well as for one of the positive wastewater samples reanalysed. As it can be seen, 4-AA eluted last, opposite to the results obtained when working with QTOF MS, where this compound eluted first. Additional experiments were performed in order to clarify if the change in elution order was due to the different column or to the different mobile phase additives. Both columns (HSS T3 and BEH C18) were tested using only $0.01 \%$ formic acid, or $0.01 \%$ formic acid plus $0.1-\mathrm{mM} \mathrm{NH}_{4} \mathrm{Ac}$ in the mobile phase. As shown in Fig. 5, the presence of $0.1-\mathrm{mM} \mathrm{NH}_{4} \mathrm{Ac}$ in the mobile phase seemed to be the responsible of this change. It is interesting to remark the higher retention of the analytes in the HSS T3 column (based on a $\mathrm{C}_{18}$ high strength silica stationary phase) than in the BEH C18 (based on ethylene bridged hybrid technology).

In the particular example shown in Fig. $4 \mathrm{~B}$, the presence of 4-AA was in principle thought after being detected in the initial LC-MS/MS analysis. However, when this sample was re-analysed under the new conditions, only small traces of 4-AA were detected, well resolved from the in-source fragments produced by the presence of high concentrations of 4-AAA and 4FAA. 


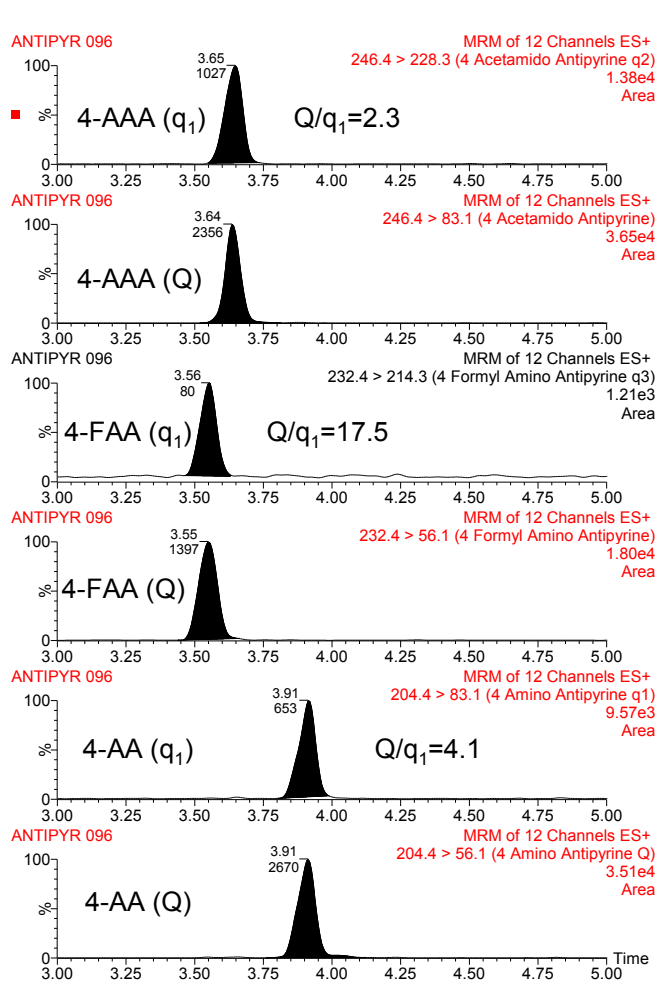

(a)

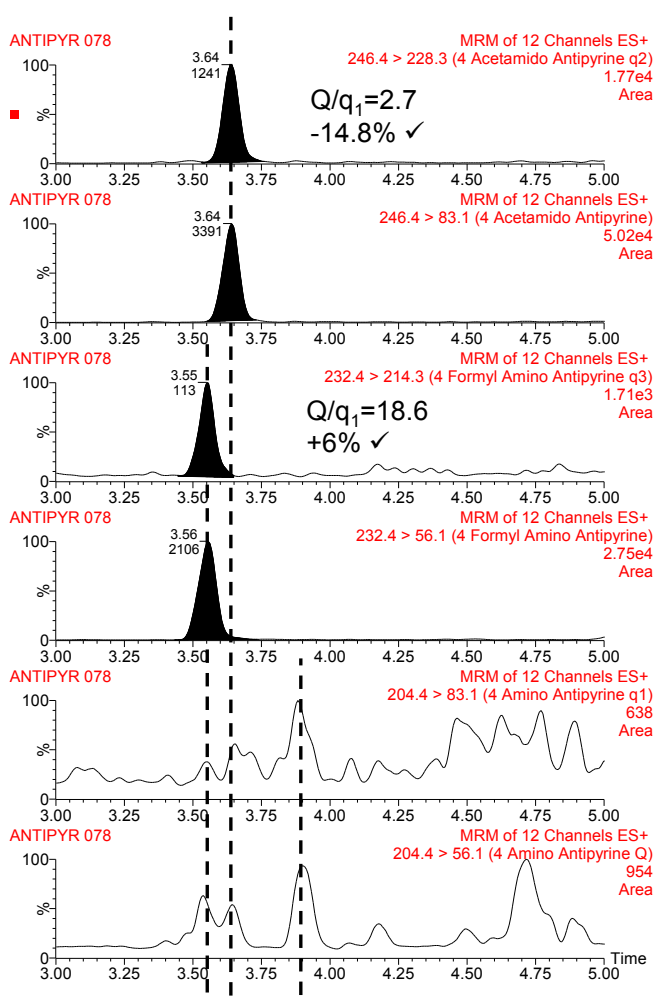

(b)

Figure 4. UHPLC-MS/MS (QqQ) chromatograms of 4-AA, 4-FAA and 4-AAA obtained for (a) $25-\mathrm{ng} / \mathrm{mL}$ reference standard and (b) wastewater sample containing 4-FAA and 4-AAA, after optimized chromatographic conditions.

After re-analysing the water samples using different chromatographic conditions and selecting specific transitions for the two additional metabolites, it was feasible to discover the presence of metabolites other than 4-AA, initially selected as the only target analyte for DIP. 4AA was now detected in only $36 \%$ of samples analysed, whereas both 4-AAA and 4-FAA were found in around $90 \%$ of the samples. 


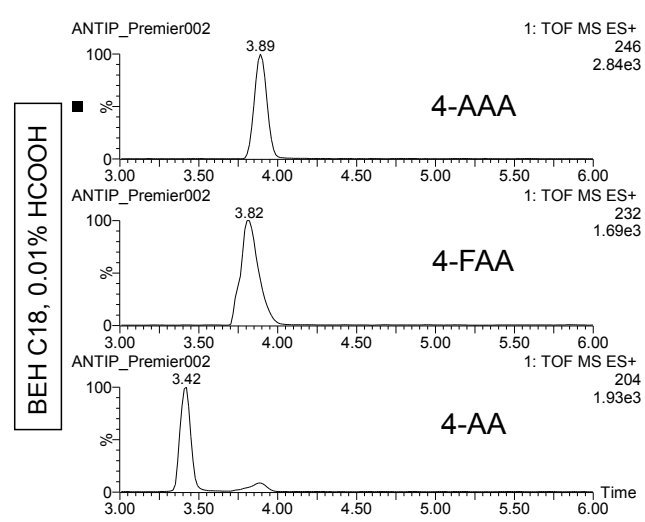

(a)
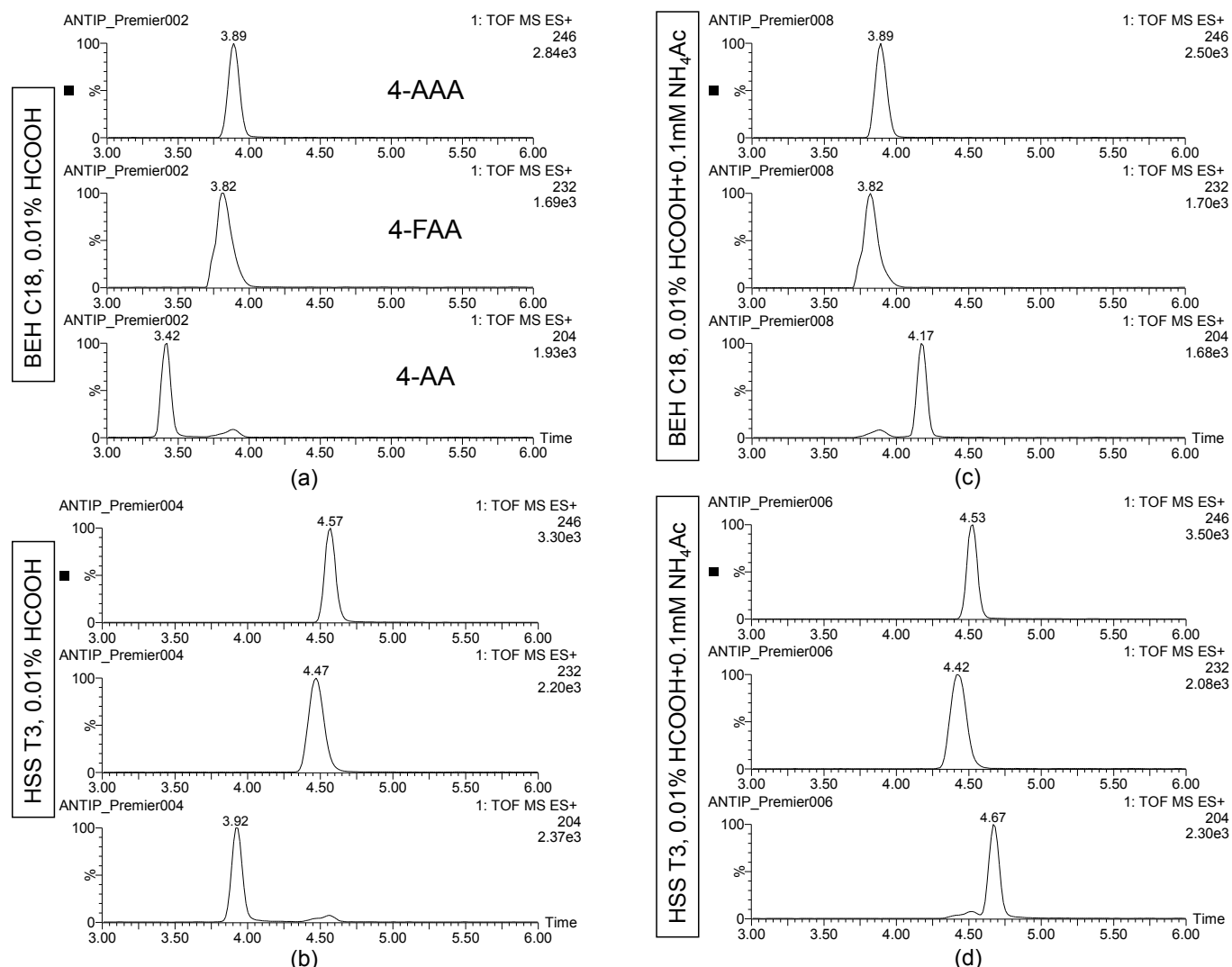

(b)

(d)

Figure 5. UHPLC-QTOF MS XICs chromatograms for a reference standard containing 4-AA, 4FAA and 4-AAA (a) BEH C18 column, using only $0.01 \%$ formic acid in the mobile phase; (b) HSS T3 column, using only $0.01 \%$ formic acid in the mobile phase; (c) BEH C18, using both $0.01 \%$ formic acid and $0.1-\mathrm{mM} \mathrm{NH}_{4} \mathrm{Ac}$ in the mobile phase. (d) HSS T3 column, using both $0.01 \%$ formic acid and $0.1-\mathrm{mM} \mathrm{NH}_{4} \mathrm{Ac}$ in the mobile phase.

The examples shown illustrate the importance of using full spectrum acquisition HRMS as well as suitable chromatographic separation to reduce the possibility of bi-isobaric co-eluting 'interferences' when investigating metabolites. These interferences might be due to unexpected metabolites that share in-source fragment ions. Surely, the problems of false identifications might have been ignored if different chromatographic conditions would have been applied, and/or if the three metabolites would have been selected as target analytes in initial analysis. However, we would like to emphasize that false positives might occur in particular situations like that described in this article, where metabolites of pharmaceuticals, and surely of many other organic contaminants like pesticides, drugs of abuse, etc. are determined even using a powerful selective technique as LC-MS/MS under SRM mode. 


\section{Conclusions}

In this work, we illustrate the problems that may occur when investigating the presence of the pro-drug DIP, selecting the metabolite 4-AA as biomarker in the LC-MS/MS analysis of environmental water. The use of HRMS helped to discover that other metabolites of DIP (e.g. 4FAA and 4-AAA) produced 4-AA via in-source fragmentation, and consequently shared the same transitions. Under these circumstances, the chromatographic separation was crucial for avoiding the assignment of erroneous identifications. This situation might also occur with other contaminants when investigating metabolites or degradation products. The analyst has to take into account that metabolites of a given compound may share the same fragment ions complicating the right identification of the compound actually present in water.

\section{Acknowledgements}

This work has been developed under financial support provided by the Spanish Ministry of Education and Science (Project reference: CTQ2009-12347). The authors also acknowledge the financial support of Generalitat Valenciana, as research group of excellence PROMETEO/2009/054. E. Gracia-Lor acknowledges University Jaume I for her pre-doctoral grant. We wish to thank WATERS and Serveis Centrals d'Instrumentació Científica of University Jaume I for using the Xevo G2 QTOF and the TQD spectrometers, respectively.

\section{References}

[1] S. D. Richardson. Water analysis: emerging contaminants and current issues. Anal. Chem. 2009, 81, 4645.

[2] S. D. Richardson. Water Analysis: Emerging Contaminants and Current Issues. Anal. Chem. 2007, 79, 4295

[3] M. Petrović, M. D. Hernando, M. S. Díaz-Cruz, D. Barceló. Liquid chromatographytandem mass spectrometry for the analysis of pharmaceutical residues in environmental samples: a review. J. Chromatogr. A 2005, 1067, 1.

[4] M. Ibáñez, C. Guerrero, J. V. Sancho, F. Hernández. Screening of antibiotics in surface and wastewater samples by ultra-high-pressure liquid chromatography coupled to hybrid quadrupole time-of-flight mass spectrometry. J. Chromatogr. A 2009, 1216, 2529.

[5] SANCO/2007/3131, Method validation and quality control procedures for pesticide residues analysis in food and feed.

[6] European Union Decision 2002/657/EC 17 August 2002. Off. J. Eur. Commun. 2002, L221, 8. 
[7] M. Ibáñez, J. V. Sancho, D. McMillan, R. Rao, F. Hernández. Rapid nontarget screening of organic pollutants in water by ultraperformance liquid chromatography coupled to time-oflight mass spectrometry. TrAC-Trends Anal. Chem. 2008, 27, 481.

[8] R. Díaz, M. Ibáñez, J. V. Sancho, F. Hernández. Target and non-target screening strategies for organic contaminants, residues and illicit substances in food, environmental and human biological samples by UHPLC-QTOF-MS. Anal. Methods 2012, 4, 196.

[9] R. Pinhancos, S. Maass, D. M. Ramanathan. High-resolution mass spectrometry method for the detection, characterization and quantitation of pharmaceuticals in water. J. Mass Spectrom. 2011, 46, 1175.

[10] A. A. M. Stolker, W. Niesing, E. Hogendoorn, J. F. M. Versteegh, R. Fuchs, U. A. T. Brinkman. Liquid chromatography with triple quadrupole or quadrupole-time of flight mass spectrometry for screening and confirmation of residues of pharmaceuticals in water. Anal. Bioanal. Chem. 2004, 378, 955.

[11] M. Petrovic, M. Gros, D. Barceló. Multi-residue analysis of pharmaceuticals in wastewater by ultra-performance liquid chromatography-quadrupole-time-of-flight mass spectrometry. J. Chromatogr. A 2006, 1124, 68.

[12] M. J. Martínez Bueno, A. Agüera, M. J. Gómez, M. D. Hernando, J. F. García-Reyes, A. R. Fernández-Alba. Application of liquid chromatography/quadrupole-linear ion trap mass spectrometry and time-of-flight mass spectrometry to the determination of pharmaceuticals and related contaminants in wastewater. Anal. Chem. 2007, 79, 9372.

[13] F. Hernández, M. Ibáñez, E. Gracia-Lor, J. V. Sancho. Retrospective LC-QTOF-MS analysis searching for pharmaceutical metabolites in urban wastewater. J. Sep. Sci. 2011, $34,3517$.

[14] M. Levy, E. Zylber-Katz, B. Rosenkranz. Clinical pharmacokinetics of dipyrone and its metabolites. Pharmacokinet. 1995, 28, 216.

[15] L. Penney, C. Bergeron, B. Coates, A. Wijewickreme. Simultaneous Determination of Residues of Dipyrone and Its Major Metabolites in Milk, Bovine Muscle, and Porcine Muscle by Liquid Chromatography/Mass Spectrometry. JAOAC Int. 2005, 88, 496.

[16] M. J. Gómez, C. Sirtori, M. Mezcua, A. R. Fernández-Alba, A. Agüera. Photodegradation study of three dipyrone metabolites in various water systems: identification and toxicity of their photodegradation products. Water Res. 2008, 42, 2698.

[17] M. J. Gómez, M. M. Gómez-Ramos, O. Malato, M. Mezcua, A. R. Fernández-Alba. Rapid automated screening, identification and quantification of organic micro-contaminants and their main transformation products in wastewater and river waters using liquid chromatography-quadrupole-time-of-flight mass spectrometry with an accurate-mass database. J. Chromatogr. A 2010, 1217, 7038.

[18] E. Gracia-Lor, J. V. Sancho, F. Hernández. Multi-class determination of around 50 pharmaceuticals, including 26 antibiotics, in environmental and wastewater samples by ultra-high performance liquid chromatography-tandem mass spectrometry. J. Chromatogr. A 2011, 1218, 2264. 
[19] T. Ternes, M. Bonerz, T. Schmidt. Determination of neutral pharmaceuticals in wastewater and rivers by liquid chromatography- electrospray tandem mass spectrometry. J. Chromatogr. A 2001, 938, 175.

[20] T. Heberer. Occurrence fate, and removal of pharmaceutical residues in the aquatic environment: a review of recent research data. Toxicol. Lett. 2005, 131, 5.

[21] B. Kasprzyk-Hordern, R. M. Dinsdale, A. J. Guwy. Multiresidue methods for the analysis of pharmaceuticals, personal care products and illicit drugs in surface water and wastewater by solid-phase extraction and ultra performance liquid chromatography-electrospray tandem mass spectrometry. Anal. Bioanal. Chem. 2008, 391, 1293.

[22] R. Díaz, M. Ibáñez, J. V. Sancho, F. Hernández. Building an empirical mass spectra library for screening of organic pollutants by ultra-high pressure liquid chromatography/hybrid quadrupole time-of-flight mass spectrometry. Rapid Commun. Mass Spectrom. 2011, 25, 355.

[23] F. Hérnández, L. Bijlsma, J. V. Sancho, R. Díaz, M. Ibáñez. Rapid wide-scope screening of drugs of abuse, prescription drugs with potential for abuse and their metabolites in influent and effluent urban wastewater by ultrahigh pressure liquid chromatography. Anal. Chim. Acta 2011, 684, 96.

[24] Y. Q. Xia, J. Lau, T. Olah, M. Jemal. Targeted quantitative bioanalysis in plasma using liquid chromatography/high-resolution accurate mass spectrometry: an evaluation of global selectivity as a function of mass resolving power and extraction window, with comparison of centroid and profile modes. Rapid Commun. Mass Spectrom. 2011, 25, 2863.

[25] F. Hernández, J. V. Sancho, M. Ibáñez. Use of UPLC-(Q)TOF MS for rapid screening of organic micro pollutants in water. In Liquid chromatography time-of flight mass spectrometry. Principles, tools and applications for accurate mass analysis, Vol. 173, I. Ferrer, E. M. Thurman (Eds). A John Wiley \& Sons, Inc, 2009

[26] A. Müller, W. Schulz, W. K. L. Ruck, W. H. Weber. A new approach to data evaluation in the non-target screening of organic trace substances in water analysis. Chemosphere 2011, 85, 1211. 